\title{
Reliability of a new test food to assess masticatory function
}

\author{
Laura Khoury-Ribas $^{\mathrm{a}, \mathrm{b}}$, Raul Ayuso-Montero ${ }^{\mathrm{a}, \mathrm{b}}$, Bernat Rovira-Lastra ${ }^{\mathrm{a}, \mathrm{b}}$, Maria Peraire ${ }^{\mathrm{a}, \mathrm{b}}$, \\ Jordi Martinez-Gomis. ${ }^{\mathrm{a}, \mathrm{b}}$, * \\ ${ }^{\text {a }}$ Department of Prosthodontics, School of Dentistry, Faculty of Medicine and Health Sciences, University of Barcelona, Catalonia, Spain \\ ${ }^{\mathrm{b}}$ Oral Health and Masticatory System Group (Bellvitge Biomedical Research Institute) IDIBELL, L'Hospitalet de Llobregat, Barcelona, Catalonia, Spain
}

\section{A R T I C L E INFO}

\section{Keywords:}

Test food

Mechanical properties

Condensation silicone

Chewing

Construct validity

\begin{abstract}
A B S T R A C T
Objective: This study assessed the reliability and validity of masticatory function assessment using a new test food, Optozeta.

Design: Thirty-five adults participated in the cross-sectional clinical part of the study; ten of them performed a retest. They performed two free-style masticatory tests consisting of five trials of 20 cycles each chewing three pieces of Optosil or Optozeta placed in a latex bag. Optozeta was created by mixing 50\% Optosil with 50\% of Zetalabor. Masticatory performance, masticatory laterality and chewing rate were assessed. Reliability and construct validity were assessed using the intraclass correlation coefficient (ICC) and Spearman correlations, respectively.

Results: Higher ICC values were observed for each aspect of masticatory function as assessed using Optozeta compared with Optosil. All the participants showed a lower median particle size value using Optozeta than Optosil. For each masticatory parameter, a high correlation was observed between using Optosil or Optozeta.

Conclusions: Optozeta seems to have good construct validity and appears to be more reliable than Optosil as a test food to assess masticatory function.
\end{abstract}

\section{Introduction}

Restoration or improvement of masticatory function is a major objective of prosthodontic treatment (Jokstad \& Orstavik, 1998). Masticatory performance, a principal aspect of this function, can be determined by quantifying the degree of fragmentation of a test food after a fixed number of chewing cycles (Flores-Orozco, Rovira-Lastra, Willaert, Peraire, \& Martinez-Gomis, 2016; Lujan-Climent et al., 2008; Van Der Bilt \& Fontijn-Tekamp, 2004). Although natural foods have been used for this, dental silicone (Optosil ${ }^{\circledR}$ ) is considered a more appropriate test food when assessing masticatory performance because it can be standardized, does not dissolve in water, and can be stored for 7 days without losing its mechanical properties (Albert, Buschang, \& Throckmorton, 2003; Compagnon, Veyrune, Morenas, \& Faulks, 1999; Edlund, 1980; Slagter, Olthoff, Bosnian, \& Steen, 1992). However, subjects wearing complete dentures, removable partial dentures, or implant-supported overdentures have been reported to have bite forces of 55-120 N, $175 \mathrm{~N}$, and $200 \mathrm{~N}$, respectively, which differ significantly from the $500 \mathrm{~N}$ reported for dentate subjects (Fontijn-Tekamp, Slagter, van't Hof, Geertman, \& Kalk, 1998; Lujan-Climent et al., 2008; Miyaura et al., 2000). Therefore, people with chewing difficulties may not comminute the Optosil pieces, which makes this artificial test food unsuitable (Slagter, Bosman, \& Van der Bilt, 1993). Optocal, an alternative chewable material composed of condensation silicone, toothpaste, solid Vaseline ${ }^{\circledR}$, dental plaster, alginate powder, and mint essence, has been described for assessing masticatory performance in this patient group (Pocztaruk, Frasca, Rivaldo, Fernandes, \& Gavião, 2008; Slagter, Bosman, \& Van der Bilt, 1993). However, the number of components and the diversity of origin make Optocal difficult to standardize. Although hardness and tensile strength have been reported for several synthetic test foods based on condensation silicone with different percentages of silicone oil, none of these alternative test foods has made a major impact in clinical studies (Compagnon, Veyrune, Morenas, \& Faulks, 1999).

Another important aspect of oral function is masticatory laterality, or masticatory jaw movements, and this can also be assessed using pieces of the artificial test food Optosil placed either in a latex bag or used freely (Farias Gomes, Custodio, Moura Jufer, Del Bel Cury, \& Rodrigues Garcia, 2010; Flores-Orozco and Tiznado-Orozco et al.,

\footnotetext{
* Corresponding author at: Campus de Bellvitge, Universitat de Barcelona, C/Feixa llarga s/n, 08907, L'Hospitalet de Llobregat, Catalonia, Spain. Email address: jmartinezgomis@ub.edu, jmartinezgomis@gmail.com (J. Martinez-Gomis.)
} 
2016; Martinez-Gomis et al., 2009; Rovira-Lastra, Flores-Orozco, Ayuso-Montero, Peraire, \& Martinez-Gomis, 2016; Rovira-Lastra, Flores-Orozco, Salsench, Peraire, \& Martinez-Gomis, 2014). To assess masticatory function in a population with compromised mastication, the ideal test food would be less elongated at its breaking point than Optosil (Compagnon, Veyrune, Morenas, \& Faulks, 1999; Slagter, Olthoff, Bosnian, \& Steen, 1992). Zetalabor is a harder condensation silicone that seems to be easily fragmented into small pieces. A new test food is therefore proposed here called Optozeta, created by mixing $50 \%$ Optosil with $50 \%$ Zetalabor, as it could be useful for assessing masticatory function in subjects wearing dental prostheses or who have difficulties chewing. The ideal method to assess different aspects of masticatory function will simple, reliable and valid (Mokkink et al., 2010).

The main objective of this study was to compare the reliability of masticatory performance assessment using Optozeta or Optosil as the test food. We also aimed to assess the reliability of masticatory laterality and chewing rate measurements using Optozeta as a test food, and to assess the construct validity with regard to masticatory performance, masticatory laterality and chewing rate using Optozeta as a test food. A preliminary study was aimed to compare hardness and elongation at breaking point of Optozeta with those of Optosil over the first 7 days after preparation. The null hypothesis was that the reliability of masticatory performance assessment using Optozeta is no different from that using Optosil.

\section{Materials and methods}

This study consisted of 2 parts: an in vitro and preliminary study to assess the mechanical properties of Optozeta and a clinical study to validate the new test food as a means of assessing masticatory function (Fig. $1)$.

In the in vitro study, 33 specimens of Optosil (Optosil P Plus; Heraeus Kulzer, Hanau, Germany) and 33 specimens of Optozeta were prepared. Of these, 18 specimens per group were dumbbell-shaped (120 mm long, $30 \mathrm{~mm}$ wide, and $2 \mathrm{~mm}$ thick) and 15 specimens per group were shaped into tablets $(20 \mathrm{~mm}$ diameter and $5 \mathrm{~mm}$ thick). These specimens were randomly assigned to groups A, B, and C, which were tested after $4 \mathrm{~h}, 3$ days, and 7 days of processing, respectively.
The putty and paste components of Optosil were mixed following the protocol described by Albert, Buschang, and Throckmorton, (2003). Briefly, one level scoop of the putty (15.5 g) was mashed vigorously with paste-hardener ( $3 \mathrm{~cm}$ long by $2 \mathrm{~mm}$ wide, $0.20 \mathrm{~g}$ in weight) for $30 \mathrm{~s}$, before being placed on a metallic base and squashed with a metallic cover separated by stops measuring $5 \mathrm{~mm}$. The resultant Optosil plates was allowed to set for $15 \mathrm{~min}$ and was then removed from the metallic base, and 15 tablets were obtained from it. The dumbbell-shaped Optosil specimens were prepared using the same protocol as for the Optosil tablets, but with six silicone sheets measuring $2 \mathrm{~mm}$ thick. Optozeta was formed following the same protocol described for Optosil, modified as follows: one researcher vigorously mashed the Optosil putty with the paste-hardener, while another researcher vigorously mashed one level scoop (17.25 g) of the Zetalabor putty component (Zhermack SPA, Rovigo, Italy) with paste-hardener ( $3 \mathrm{~cm}$ long by $2 \mathrm{~mm}$ wide, $0.20 \mathrm{~g}$ in weight), both for $10 \mathrm{~s}$; then, one of the researchers combined the two silicone mixtures and vigorously mashed them for a further $20 \mathrm{~s}$. Shore A hardness was measured at the three study time points $(\mathrm{n}=5)$, using a durometer in accordance with ISO 868.2003. Tensile testing was conducted in accordance with ISO 37-1 and the crosshead speed was $500 \mathrm{~mm} / \mathrm{min}$. Elongation at breaking point were recorded for the two silicones at the three time points $(n=6)$ (Fig. 1).

In the cross-sectional clinical study, 35 adults (23 women and 12 men; age range: 19-77 years, mean age 37), were recruited from students and staff at the University of Barcelona Dental School (Catalonia, Spain) and from patients attending the Barcelona University Dental Hospital. Of the participants, 25 had natural dentition, 6 wore removable partial or complete dentures, and 4 wore implant-supported partial prostheses (Fig. 1). A test-retest was performed on 15 participants (11 women, mean age 34, 13 had natural dentition and 2 wore removable dentures), chosen by convenience, 1-2 weeks after the first test. The subjects were fully informed and signed an informed consent form approved by the Barcelona University Dental Hospital Ethics Committee (Code 2015/32). All the experiments were carried out in accordance with the principles of the Helsinki Declaration.

Each participant performed two different masticatory assays each consisting of five trials of 20 cycles each chewing $2 \mathrm{~g}$ of silicon. Optosil and Optozeta tablets ( $5 \mathrm{~mm}$ thick, $20 \mathrm{~mm}$ diameter) were produced as

\section{In vitro study}

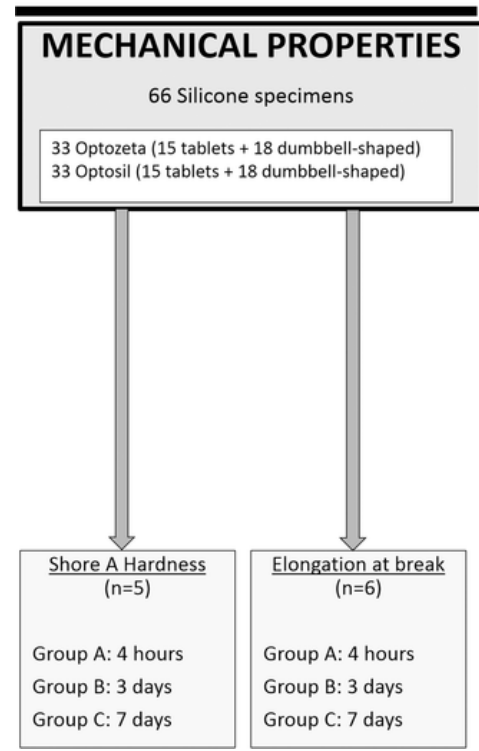

Clinical study

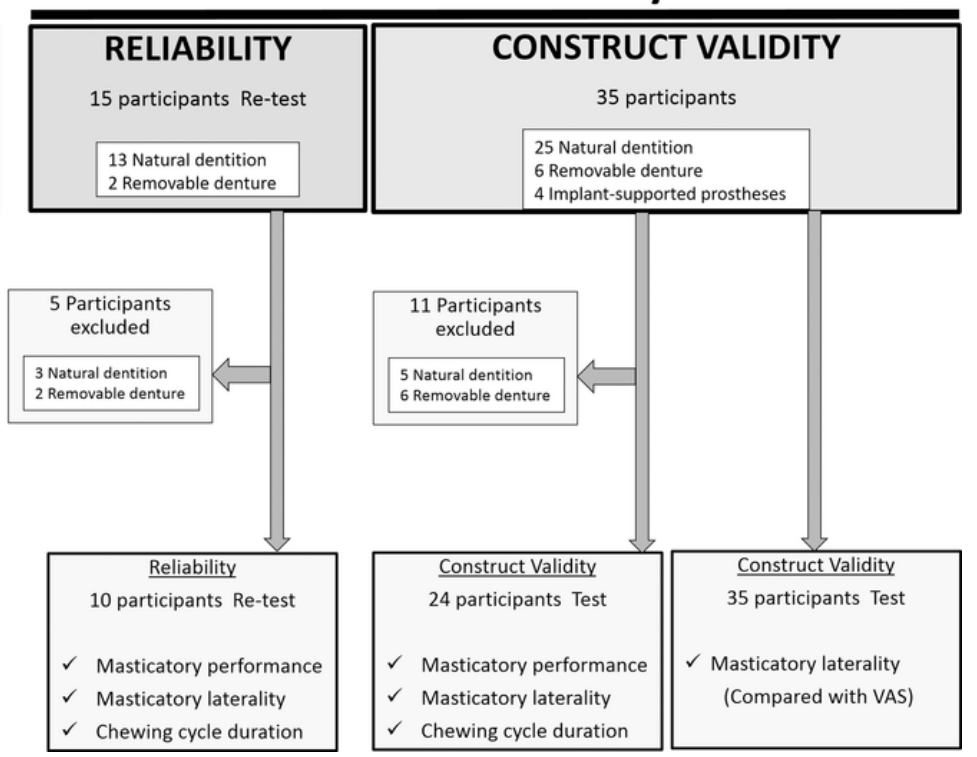

Fig. 1. Flowchart of the in vitro and clinical studies. 
for the in vitro study, cut into quarters, and three of the quarter tablets were placed in a latex bag which was then sealed (Rovira-Lastra et al., 2014). The two assays consisted of free-style mastication: in one, the subjects chewed the bagged Optosil test food and in the other, the bagged Optozeta, and the sequence was randomised. Participants were not aware of the type of silicone chewed in the assays.

Masticatory performance was evaluated for each masticatory assay by assessing the degree of comminution of the silicon test food (Lujan-Climent et al., 2008). For each assay, the particles from five trials $(10 \mathrm{~g})$ were dried for $24 \mathrm{~h}$ and passed through a series of eight sieves $(0.25,0.425,0.85,2,2.8,3.15,4$, and $5.6 \mathrm{~mm})$ while being shaken for $1 \mathrm{~min}$. After the cumulative weight distribution of the sieve contents had been determined, the median particle size (MPS) was calculated for each subject using the Rosin-Rammler equation [Qw $(\mathrm{X})=1$ - $2 \mathrm{E}-\left(\mathrm{X} / \mathrm{X}_{50}\right)^{\mathrm{b}}$ ], where $\mathrm{Qw}(\mathrm{X})$ is the fraction of particles by weight with a diameter smaller than $\mathrm{X}$; $\mathrm{X}_{50}$ (or MPS) is the size of a theoretical sieve through which $50 \%$ of the weight would pass; and b describes the breadth of the particle size distribution (Olthoff, van der Bilt, \& Bosman, 1984). Therefore, the lower the MPS, the better the masticatory performance. The total duration of each of the five trials was used to calculate the duration of the average chewing cycle for each masticatory assay (Salsench et al., 2005). A video camera (Sony HDR-UX7E, Tokyo, Japan) recorded mandible displacement while closing during each mastication assay. The preferred chewing side was determined by three methods. The first method (PCS_1st cycle) was based on the definition as "the mandibular side favoured during the closing phase for the first masticatory cycle" (Hoogmartens \& Caubergh, 1987), measured five times using an index as follows: $\mathrm{I}=$ (right - left $) /($ right + left $)$. The second method (PCS_All cycles) used all the masticatory cycles to calculate the asymmetry index (AI), as follows: $\mathrm{AI}=$ (number of right strokes - number of left strokes)/(number of right strokes + number of left strokes) (Mizumori, Tsubakimoto, Iwasaki, \& Nakamura, 2003). The last method (PCS_VAS) consisted of using a VAS assessment in which the subjects made a mark on a $10 \mathrm{~cm}$ line with 'always left' $(-1)$ and 'always right' $(+1)$ at either end, and 'no preference' $(0)$ in the middle (Flores-Orozco, Rovira-Lastra and Peraire et al., 2016).

The sample size for the main objective was calculated considering a type I error of 0.05 and a power of 0.8 in order to find an ICC of 0.9 , a $95 \%$ CI of 0.2 and a test-retest of 2 time points (Lujan-Climent et al., 2008). The sample size to assess the construct validity using Optozeta was determined accepting a type I error of 0.05 and a power of 0.2 in a two-sided test, an estimated drop-out rate of $15 \%$, with a correlation coefficient of 0.5 (Flores-Orozco, Rovira-Lastra and Peraire et al., 2016). Two-way analysis of variance (ANOVA) was used for the analysis of hardness (type of test food and time after processing). Then the Duncan post hoc test was used to determine differences between the groups. The error variance of the analysis of the elongation at breaking point (as a dependent variable) was unequal between the study groups, so the Mann-Whitney $U$ test was used for the analysis. Test-retest reliability was assessed by the intraclass correlation coefficient (ICC) for masticatory parameters using a mixed model with a random effect for the individual. Spearman correlations and the Wilcoxon test were performed to assess the construct validity with regard to masticatory parameters using Optozeta or Optosil as the test food; or with VAS for masticatory laterality. P-values below 0.05 were considered statistically significant, and all the analysis was conducted using the SPSS program (IBM SPSS Statistics, version 23.0.0.2, Chicago, IL).

\section{Results}

The two-way ANOVA showed that time after processing and the type of silicone affected hardness $(\mathrm{P}<0.001)$, and that there was a significant interaction between these two variables $(\mathrm{P}=0.02)$. Thus, the hardness of Optozeta was greater than that of Optosil alone, with Optosil showing a faster increase in hardness than Optozeta (Fig. 2). Although there was no statistically significant difference $(\mathrm{P}=0.06$; Mann-Whitney $U$ test), Optozeta tolerated a $30 \%$ elongation without breaking and Optosil tolerated a 36\% elongation without breaking. Moreover, the standard deviations for Optosil were larger than those for Optozeta (Fig. 3).

To assess the test-retest reliability of the masticatory performance using Optozeta or Optosil as the test food, participants were excluded if their MPS was higher than $10.6 \mathrm{~mm}$ (a piece of silicone without being

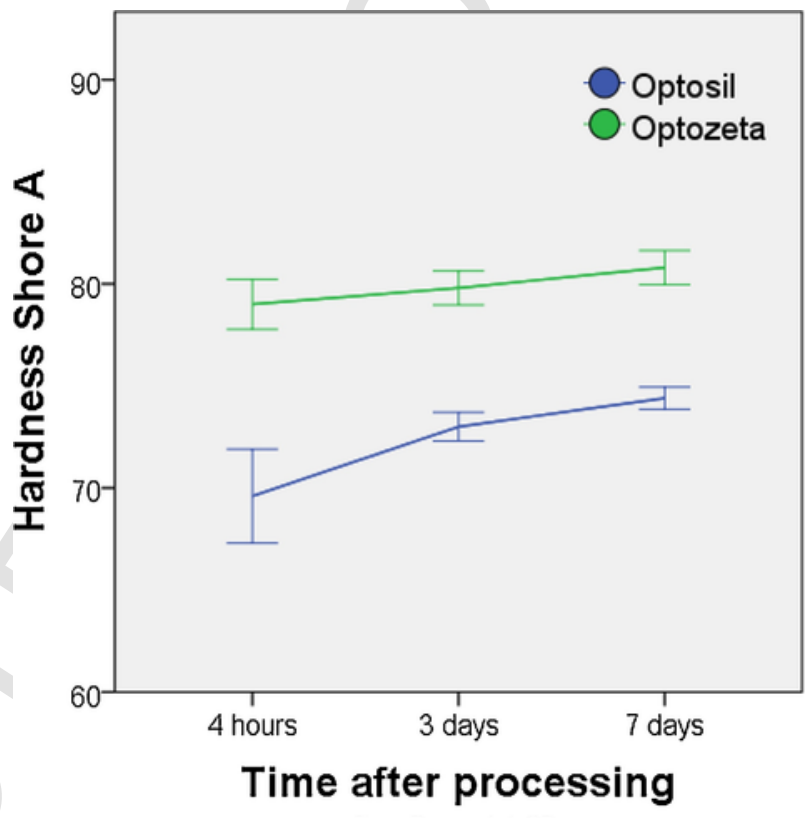

Error Bars: $+/-1 \mathrm{SD}$

Fig. 2. Hardness of Optosil and Optozeta at different times after setting $(n=5)$. Time after processing and the type of silicone affected hardness $(\mathrm{P}<0.001$; Two-way ANOVA)

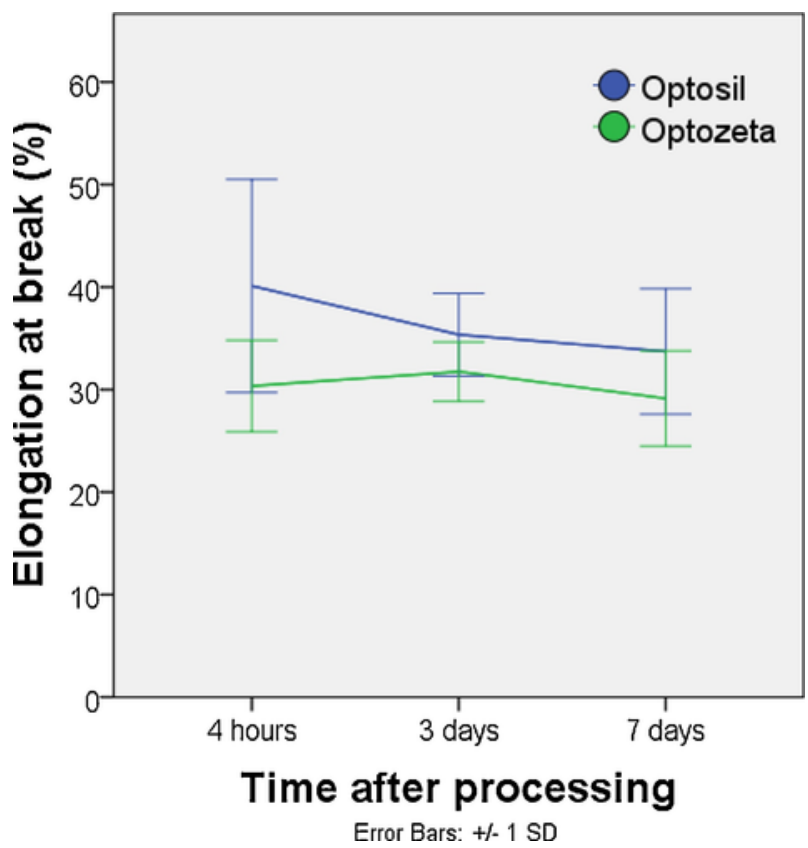

Fig. 3. Elongation at breaking point of Optosil and Optozeta at different times after setting $(n=6)$. No significant differences between the type of silicone at each time point (P > 0.05; Mann-Whitney U Test). 
broken down would pass through a $10.6 \mathrm{~mm}$ sieve; therefore an MPS greater than $10.6 \mathrm{~mm}$ has no sense). Of the 15 participants in the retest, five participants ( 3 with natural dentition and 2 wearing a removable denture) had an MPS greater than $10.6 \mathrm{~mm}$ in either the test or retest with Optosil and therefore were excluded (Fig. 1). However, two of the excluded participants had MPS less than $10.6 \mathrm{~mm}$ in both the test and retest with Optozeta as the test food. Higher ICC values for each aspect of masticatory function assessed using Optozeta was observed, compared to those using Optosil (Table 1).

To assess the construct validity with regard to masticatory performance, among 35 participants eleven ( 6 with removable dentures and 5 with natural dentition) were excluded because their MPS obtained with Optosil was more than $10.6 \mathrm{~mm}$ (Fig. 1). For each masticatory parameter, a high correlation was observed between using Optosil or Optozeta as the test food (Table 2 and Figs. 4-6). All the participants showed a smaller MPS using Optozeta (median $3.0 \mathrm{~mm}$ ) than with Optosil (median $4.4 \mathrm{~mm}$ ) (Table 2 and Fig. 4). However, masticatory laterality and chewing cycle duration were not significantly different whether the participants were chewing Optosil or Optozeta (Table 2 and Figs. 5 and 6). Table 3 shows the correlation matrix between the different methods of assessing the preferred chewing side without excluding any participant. A similar correlation was found between preferred chewing side determined with VAS and Optosil compared to VAS and Optozeta.

\section{Discussion}

The main finding of this study was that assessing masticatory performance using Optozeta as the test food is more reliable than doing with Optosil; therefore, the null hypothesis was rejected. This was corroborated in the clinical study, in which Optozeta showed better discrimination between participants and better agreement between sessions; not only for masticatory performance, but also for masticatory laterality and chewing rate. Furthermore, all the participants had a smaller MPS using Optozeta than with Optosil and three of them would not have been excluded taken into account the MPS values using Optozeta. Moreover, the scores for each aspect of masticatory function obtained using Optozeta as the test food were consistent with scores of masticatory function obtained using Optosil, thereby demonstrating a good construct validity of Optozeta as a test food to assess masticatory function.

The masticatory performance of most of the participants with natural dentition or wearing implant-supported prostheses was assessed adequately using Optozeta. However, 4 of the 6 participants with removable dentures were not assessed adequately with Optozeta. This could be explained by the lower bite force of these patients or the fact that these patients wore removable dentures with defects. It is known that people who wear removable dentures have approximately 2- to 6-times less bite force than dentate adults (Fontijn-Tekampl et al., 1998; Miyaura, Morita, Matsuka, Yamashita, \& Watanabe, 2000). Moreover, masticatory performance depends not only on bite force and occlusal contact area (Lujan-Climent et al., 2008), but also on the status of the dentures (Asakawa, Fueki, \& Ohyama, 2005; Gambareli, Serra, Pereira, \& Gavião, 2007; Martori et al., 2017; Martori, Ayuso-Montero,
Martinez-Gomis, Viñas, \& Peraire, 2014). In fact, most of these patients sought treatment related to defects of their denture, such as worn acrylic teeth or lack of denture retention. Optocal, an alternative chewable material composed of condensation silicone, toothpaste, solid Vaseline ${ }^{\circledR}$, dental plaster, alginate powder, and mint essence, could be used to assess masticatory performance in people with chewing difficulties (Pocztaruk, Frasca, Rivaldo, Fernandes, \& Gavião, 2008; Slagter, Bosman, \& Van der Bilt, 1993). However, the large number of components and the diversity of its origin make it difficult to standardize and store the Optocal pieces.

The mechanical properties of the new test food showed that energy input was more likely to cause it to break than simply to deform. Therefore, although a slightly greater force was needed to indent it than was needed for Optosil, similar force would break Optozeta into smaller particles. The mechanical properties of Optozeta were more stable for the first 7 days after setting than those of Optosil. Furthermore, the intra-group variability was lower with Optozeta than for Optosil. Therefore, storage for 7 days appears acceptable because no excessive changes were observed in the mechanical properties.

The mechanical properties of different types of silicone have been compared with those of different natural foods (Compagnon, Veyrune, Morenas, \& Faulks, 1999; Slagter, Olthoff, Bosnian, \& Steen, 1992). However, it is very difficult to provide any consistent equivalence between the mechanical properties of a natural food and those of an artificial test food because of the wide variability in the mechanical properties of natural foods (Edlund, 1980). Further studies are needed to demonstrate equivalence between artificial test foods and natural foods.

The Masticatory Normative Indicator, based on the median particle size before swallowing, is considered a valid discriminator between normal and impaired masticatory function using carrots or Optosil as a test food (Woda et al., 2010; Witter, Woda, Bronkhorst, \& Creugers, 2013). However, this indicator gives an idea of masticatory efficacy regardless of the number of chewing cycles used to comminute the food. It would be interesting to know the cut-off of MPS that reflects the normal masticatory performance using the Optozeta as a test food and thereby to establish the Masticatory Performance Normal Indicator. This could be the aim of further study in a larger sample.

Masticatory laterality has also been assessed using various test foods, including chewing gum, natural foods, and artificial test foods. Optosil (placed in a latex bag or loose) has been demonstrated to be a reliable test food for assessing this function in adults with natural dentition (Farias Gomes et al., 2010; Flores-Orozco and Tiznado-Orozco et al., 2016; Flores-Orozco, Rovira-Lastra and Willaert et al., 2016; Martinez-Gomis et al., 2009; Rovira-Lastra et al., 2014; Rovira-Lastra et al., 2016). The results of the present study demonstrate that Optozeta could not only be suitable for assessing masticatory laterality in dentate subjects but also in people with chewing difficulties or wearing dental prostheses.

One of the limitations of the present study is that reliability was measured in individuals with natural dentition and, thus only applicable on this population. However, the masticatory function of the four patients with implant-supported prosthesis was similar to the individuals with natural dentition with good masticatory performance. There-

Table 1

Test-retest reliability of masticatory performance, masticatory laterality, and chewing rate assessed using Optosil or Optozeta as the test food.

\begin{tabular}{|c|c|c|c|c|}
\hline & \multirow[t]{2}{*}{ Median particle size ICC (CI95\%) } & \multicolumn{2}{|c|}{ Preferred Chewing Side ICC (CI95\%) } & \multirow[t]{2}{*}{ Chewing cycle duration ICC (CI95\%) } \\
\hline & & First cycle & All cycles & \\
\hline Optosil & $0.62(0.06-0.89)$ & $0.64(0.05-0.90)$ & $0.79(0.38-0.94)$ & $0.63(0.10-0.89)$ \\
\hline Optozeta & $0.67(0.10-0.91)$ & $0.92(0.73-0.98)$ & $0.90(0.64-0.97)$ & $0.76(0.29-0.93)$ \\
\hline
\end{tabular}

ICC-Intraclass coefficient correlation. $\mathrm{n}=10$. 
Table 2

Correlation and concordance of masticatory performance, masticatory laterality, and chewing rate using Optosil or Optozeta as the test food.

\begin{tabular}{|c|c|c|c|}
\hline & Correlation & & Concordance \\
\hline & $\begin{array}{l}\text { Spearman } \\
\text { coefficient }\end{array}$ & P-Value & $\begin{array}{l}\text { P-value, } \\
\text { Wilcoxon } \\
\text { test }\end{array}$ \\
\hline Median particle size & 0.807 & $<0.001$ & $<0.001$ \\
\hline $\begin{array}{l}\text { Preferred chewing side } \\
\text { (1st cycle) }\end{array}$ & 0.823 & $<0.001$ & 0.66 \\
\hline $\begin{array}{l}\text { Preferred chewing side } \\
\text { (All cycles) }\end{array}$ & 0.884 & $<0.001$ & 0.92 \\
\hline Chewing cycle duration & 0.874 & $<0.001$ & 0.38 \\
\hline
\end{tabular}

$\mathrm{n}=24$.

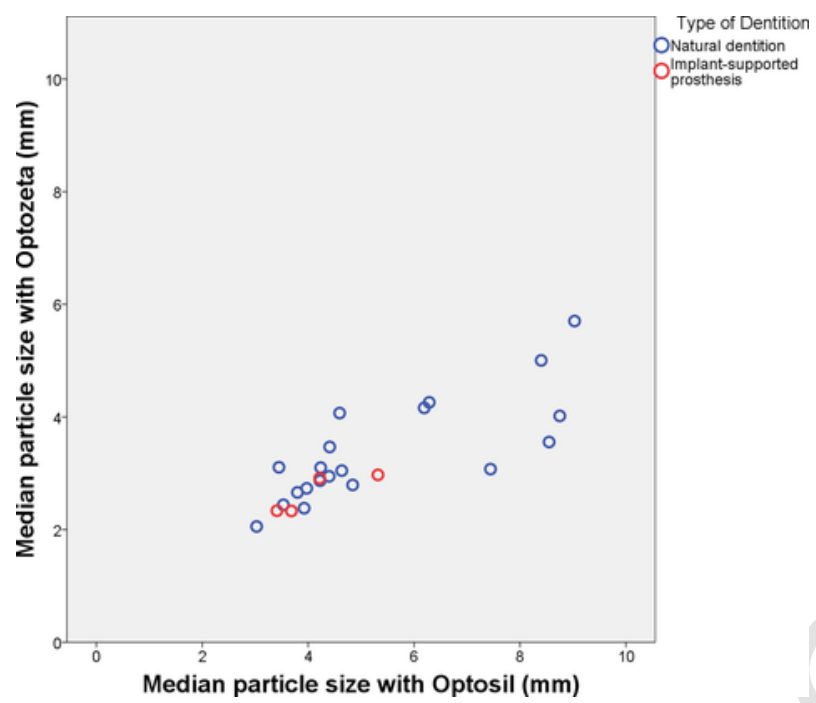

Fig. 4. Relationship between masticatory performance scores as determined using Optosil or Optozeta as the test food by the type of dentition $(n=24)$.

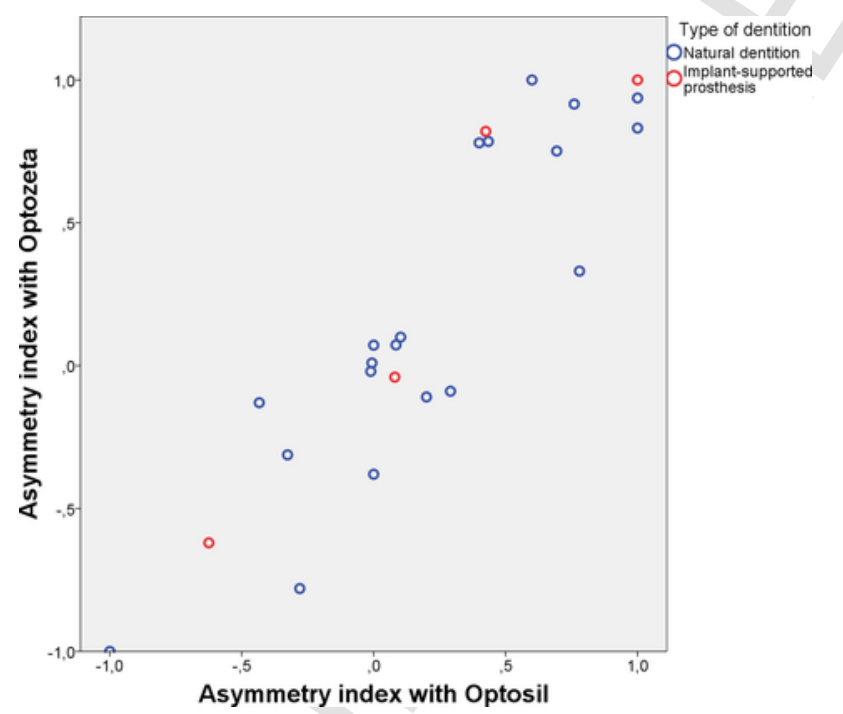

Fig. 5. Relationship between masticatory laterality assessed using Optosil or Optozeta as the test food by the type of dentition $(n=24)$.

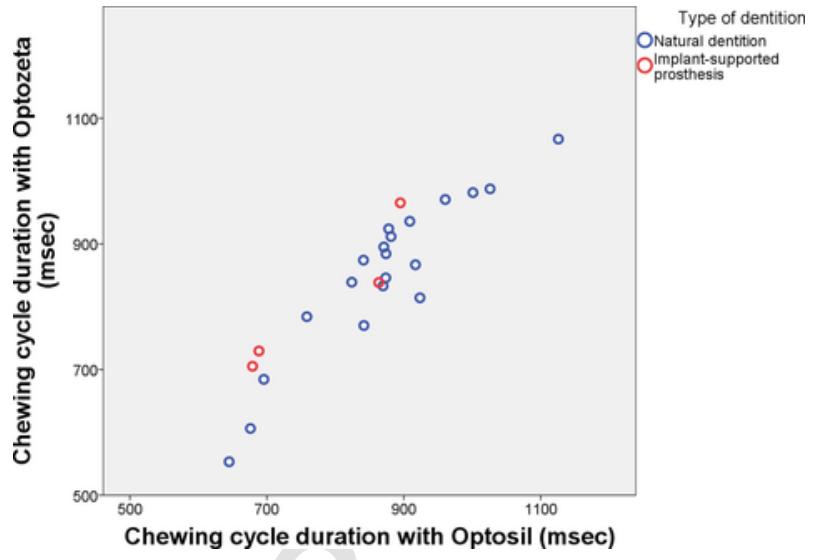

Fig. 6. Relationship between chewing rate scores obtained using Optosil or Optozeta as the test food by the type of dentition $(n=24)$.

fore, it is likely that Optozeta be a better test food than Optosil to measure masticatory function in patients with implant-supported prosthesis, but this extrapolation should be taken with caution. Another weakness of this study was the small sample size in the test-retest, and, although it was sufficient to know which test-food makes the more reliable method, it was probably insufficient to know the actual magnitude of reliability.

In conclusion, Optozeta appears to be more reliable than Optosil as a test food to assess masticatory performance, masticatory laterality and chewing rate. Optozeta seems to have good construct validity as a test food to assess masticatory function. Optozeta is harder than Optosil and it is also more stable for the first 7 days after setting.

\section{Conflict of interests}

The authors confirm that there are no known conflicts of interest associated with this publication and there has been no significant financial support for this work that could have influenced its outcome.

\section{Funding}

This research received partial funding from the Bellvitge Campus Research Committee (ACESB 2014), and from the Faculty of Dentistry, both of the University of Barcelona.

\section{Ethical Approval}

This study was approved by the Barcelona University Dental Hospital Ethics Committee (Code 2015/32).

\section{Acknowledgments}

The authors gratefully acknowledge the mechanical tests performed by Biomaterials, Biomechanics and Tissue Engineering Group, Department Materials Science and Metallurgical Engineering, ETSEIB Technical University of Catalonia (UPC). The authors would like to thank Dr Christopher Evans for editing the text. This research received partial funding from the Bellvitge Campus Research Committee (ACESB 2014), and from the Faculty of Dentistry, both of the University of Barcelona. 
Table 3

Spearman correlation coefficient matrix for the different methods used to determine preferred chewing side.

\begin{tabular}{|c|c|c|c|c|c|}
\hline & PCS (VAS) & PCS (1st cycle) Optosil & PCS (All cycles) Optosil & PCS (1st cycle) Optozeta & PCS (All cycles) Optozeta \\
\hline PCS (VAS) & 1 & & & & \\
\hline PCS (1st cycle) Optosil & $0.56(<0.001)$ & 1 & & & \\
\hline PCS (All cycles) Optosil & $0.60(<0.001)$ & $0.73(<0.001)$ & 1 & & \\
\hline PCS (1st cycle) Optozeta & $0.55(0.001)$ & $0.72(<0.001)$ & $0.62(<0.001)$ & 1 & \\
\hline PCS (All cycles) Optozeta & $0.55(0.001)$ & $0.67(<0.001)$ & $0.85(<0.001)$ & $0.71(<0.001)$ & \\
\hline
\end{tabular}

PCS - Preferred chewing side, VAS - Visual analogue scale. $\mathrm{n}=35$.

\section{References}

Albert, T.E., Buschang, P.H., Throckmorton, G.S., 2003. Masticatory performance: A protocol for standardized production of an artificial test food. Journal of Oral Rehabilitation $30,720-722$.

Asakawa, A., Fueki, K., Ohyama, T., 2005. Detection of improvement in the masticatory function from old to new removable partial dentures using mixing ability test. Journal of Oral Rehabilitation 32, 629-634.

Compagnon, D., Veyrune, J.L., Morenas, M., Faulks, D., 1999. Development of a synthetic bolus using silicone elastomer for the study of masticatory efficiency. The Journal of Prosthetic Dentistry 81, 704-709.

Edlund, J.L.C., 1980. Masticatory efficiency. Journal of Oral Rehabilitation 7, 123-130.

Farias Gomes, S.G., Custodio, W., Moura Jufer, J.S., Del Bel Cury, A.A., Rodrigues Garcia, R.C.M., 2010. Correlation of mastication and masticatory movements and effect of chewing side preference. Brazilian Dental Journal 21, 351-355.

Flores-Orozco, E.I., Tiznado-Orozco, G.E., Osuna-González, O.D., Amaro-Navarrete, C.L., Rovira-Lastra, B., Martinez-Gomis, J., 2016. Lack of relationship between masticatory performance and nutritional status in adults with natural dentition. Archives of Oral Biology 71, 117-121.

Flores-Orozco, E.I., Rovira-Lastra, B., Peraire, M., Salsench, J., Martinez-Gomis, J., 2016. Reliability of a visual analog scale for determining the preferred mastication side. The Journal of Prosthetic Dentistry 115, 203-208.

Flores-Orozco, E.I., Rovira-Lastra, B., Willaert, E., Peraire, M., Martinez-Gomis, J., 2016. Relationship between jaw movement and masticatory performance in adults with natural dentition. Acta Odontologica Scandinavica 74, 103-107.

Fontijn-Tekampl, E., Slagter, A.P., Van't Hof, M.A., Geertman, M.E., Kalk, W., 1998. Bite forces with mandibular implant-retained overdentures. Journal of Dental Research 77, 1832-1839.

Gambareli, F.R., Serra, M.D., Pereira, L.J., Gavião, M.B.D., 2007. Teeth and muscle force interactions in. Journal of Texture Studies 38, 2-20.

Hoogmartens, M.J., Caubergh, M.A.A., 1987. Chewing side preference in man correlated with handedness, footedness: Eyedness and earedness. Electromyography and Clinical Neurophysiology 27, 293-300.

Jokstad, A., Orstavik, J.R.T., 1998. A definition of prosthetic dentistry. International Journal of Prosthodontics 11, 295-301.

Lujan-Climent, M., Martinez-Gomis, J., Palau, S., Ayuso-Montero, R., Salsench, J., Peraire, M., 2008. Influence of static and dynamic occlusal characteristics and muscle force on masticatory performance in dentate adults. European Journal of Oral Sciences 116, 229-236.

Martinez-Gomis, J., Lujan-Climent, M., Palau, S., Bizar, J., Salsench, J., Peraire, M., 2009. Relationship between chewing side preference and handedness and lateral asymmetry of peripheral factors. Archives of Oral Biology 54, 101-107.
Martori, E., Ayuso-Montero, R., Martinez-Gomis, J., Viñas, M., Peraire, M., 2014. Risk factors for denture-related oral mucosal lesions in a geriatric population. The Journal of Prosthetic Dentistry 111, 273-279.

Martori, E., Ayuso-Montero, R., Willaert, E., Viñas, M., Peraire, M., Martinez-Gomis, J., 2017. Status of removable dentures and relationship with oral candida-associated factors in a geriatric population in catalonia. Journal of Prosthodontics 26, 370-375.

Miyaura, K., Morita, M., Matsuka, Y., Yamashita, A., Watanabe, T., 2000. Rehabilitation of biting abilities in patients with different types of dental prostheses. Journal of Oral Rehabilitation 27, 1073-1076.

Mizumori, T., Tsubakimoto, T., Iwasaki, M., Nakamura, T., 2003. Masticatory laterality-evaluation and influence of food texture. Journal of Oral Rehabilitation 30, 995-999.

Mokkink, L.B., Terwee, C.B., Patrick, D.L., Alonso, J., Stratford, P.W., Knol, D.L., et al., 2010. The COSMIN study reached international consensus on taxonomy, terminology, and definitions of measurement properties for health-related patient-reported outcomes. Journal of Clinical Epidemiology 63, 737-745.

Olthoff, L.W., van der Bilt, A., Bosman, F.K.H., 1984. Distribution of particle sizes in food comminuted by human mastication. Archives of Oral Biology 29, 899-903.

Pocztaruk, R.D.L., Frasca, L.C.D.F., Rivaldo, E.G., Fernandes, E.D.L., Gavião, M.B.D., 2008 Protocol for production of a chewable material for masticatory function tests (Optocal - Brazilian version). Brazilian Oral Research 22, 305-310.

Rovira-Lastra, B., Flores-Orozco, E.I., Salsench, J., Peraire, M., Martinez-Gomis, J., 2014. Is the side with the best masticatory performance selected for chewing?. Archives of Oral Biology 59, 1316-1320.

Rovira-Lastra, B., Flores-Orozco, E.I., Ayuso-Montero, R., Peraire, M., Martinez-Gomis, J., 2016. Peripheral: Functional and postural asymmetries related to the preferred chewing side in adults with natural dentition. Journal of Oral Rehabilitation 43, 279-285.

Salsench, J., Martinez-Gomis, J., Torrent, J., Bizar, J., Samso, J., Peraire, M., 2005. Relationship between duration of unilateral masticatory cycles and the type of lateral dental guidance: A preliminary study. The International Journal of Prosthodontics 18 339-346.

Slagter, A.P., Olthoff, L.W., Bosnian, F., Steen, W.H.A., 1992. Masticatory ability, denture quality: And oral conditions in edentulous subjects. The Journal of Prosthetic Dentistry 68, 299-307.

Slagter, A., Bosman, F., Van der Bilt, A., 1993. Comminution of two artificial test foods by dentate and edentulous subjects. Journal of Oral Rehabilitation 20, 159-176.

Van Der Bilt, A., Fontijn-Tekamp, F.A., 2004. Comparison of single and multiple sieve methods for the determination of masticatory performance. Archives of Oral Biology 49, 193-198.

Witter, D.J., Woda, A., Bronkhorst, E.M., Creugers, N.H., 2013. Clinical interpretation of a masticatory normative indicator analysis of masticatory function in subjects with different occlusal and prosthodontic status. Journal of Dentistry 41, 443-448.

Woda, A., Nicolas, E., Mishellany-Dutour, A., Hennequin, M., Mazille, M.N., Veyrune, J.L., et al., 2010. The masticatory normative indicator. Journal of Dental Research 89, 281-285. 